\title{
Measuring primality in numerical semigroups with embedding dimension three
}

\author{
S. T. Chapman \\ Sam Houston State University \\ Department of Mathematics and Statistics \\ Box 2206, Huntsville, TX 77341-2206 \\ scott.chapman@shsu.edu \\ P. A. García-Sánchez \\ Departamento de Álgebra and CITIC-UGR \\ Universidad de Granada \\ E-18071 Granada, España \\ pedro@ugr.es \\ Z. Tripp \\ Tufts University, Department of Mathematics \\ 503 Boston Avenue, Bromfield-Pearson \\ Room 103, Medford, MA 02155 \\ trippzac@gmail.com \\ C. Viola \\ Dipartimento di Matematica e Informatica \\ Viale A. Doria, 6 - I 95125, Catania, Italia \\ violacaterina@gmail.com \\ Received 21 August 2014 \\ Accepted 27 December 2014 \\ Published 31 August 2015 \\ Communicated by R. Wiegand
}

In this paper, we find the $\omega$-value of the generators of any numerical semigroup with embedding dimension three. This allows us to determine all possible orderings of the $\omega$-values of the generators. In addition, we relate the $\omega$-value of the numerical semigroup to its catenary degree.

Keywords: Prime element; numerical semigroup; omega function. 


\section{Introduction}

The arithmetic of non-unique factorizations in rings and monoids has been a popular topic in the recent mathematical literature. We focus on extending the results in $[3,4,8]$, where the $\omega$-function, an arithmetic measure of how far an element is from being prime (cf. Sec. 2.3), is studied in numerical semigroups. In [3], the authors present an algorithm for computing values of the omega function on any numerical semigroup, and focus on computing $\omega$-values for the generators of an embedding dimension three semigroup. When $S$ is a numerical semigroup with minimal set of generators $\left\{n_{1}, n_{2}, n_{3}\right\}$, with $n_{1}<n_{2}<n_{3}$, there are 13 possible inequalities involving the $\omega\left(n_{i}\right)$ (for instance $\omega\left(n_{1}\right) \leq \omega\left(n_{2}\right)<\omega\left(n_{3}\right)$ ). Examples of eight of these orderings were given in [3], and two more were given in [8]. The authors in [8] conjectured that the last three orderings are not possible. In this paper we compute the $\omega$-values of the generators in the embedding dimension three case (Theorems 4.6 and 4.9). As a by-product, in Theorem 6.2 we confirm the conjecture cited above in [8]. Additionally, we relate, for embedding dimension three, the $\omega$-value of a numerical semigroup to its catenary degree (cf. Sec. 2.2). We open in Sec. 2 with a brief review of definitions needed in the paper's remaining four sections. In Sec. 3, we consider the structure of the set of bullets (cf. Sec. 2.3) for the generators. In Sec. 4 , we produce the $\omega$-values for the generators in the case where the semigroup has embedding dimension three. Section 5 considers the relation between the $\omega$-function and the catenary degree and Sec. 6 discusses the resolution of the conjecture mentioned above from [8].

In addition to $[3,4,8]$ previously mentioned, we note that there has been much recent work on the behavior of the $\omega$-function in several different settings. In $[1,2]$ the behavior of the $\omega$-function is studied on commutative rings and integral domains. The software package [10] will compute values of the $\omega$-function in any affine semigroup (finitely generated submonoids of $\mathbb{N}^{k}$ ), and was used in [11] to obtain some general and asymptotic results. We note that the package [9] now deals also with affine semigroups, and has optimizations for full affine semigroups; see Chapter 10 of the manual. We used [9] for making batteries of examples that led us to many of the results presented in the current paper. It is shown recently in [20] that the omega function on a numerical semigroup is eventually quasi-linear, and the same authors in [21] give a comprehensive survey of the results in $[4,11,20]$. The current paper is mostly self-contained. Undefined terminology or notation regarding numerical semigroups or factorization theory can be found in $[15,22]$, respectively.

\section{Preliminaries}

A numerical semigroup $S$ is a nonempty subset of the set of nonnegative integers $\mathbb{N}$ that is closed under addition, contains 0 and has finite complement in $\mathbb{N}$. The condition $\#(\mathbb{N} \backslash S)<\infty$ is equivalent to $\operatorname{gcd}(S)=1$ (see for instance [22, Chap. 1]). The largest integer not belonging to $S$ is its Frobenius number $\mathrm{F}(S)$. Recall that $S$ 
is symmetric if for every $x \in \mathbb{Z} \backslash S, \mathrm{~F}(S)-x \in S$. In [22, Chap. 3] the reader can find more characterizations of the symmetric property.

We say that the positive integers $n_{1}, \ldots, n_{p}$ generate $S$ if $S=\left\{\sum_{i=1}^{p} x_{i} n_{i} \mid x_{i} \in\right.$ $\mathbb{N}$ for each $i\}$. For such a generating set, we use the notation $S=\left\langle n_{1}, \ldots, n_{p}\right\rangle$. Using elementary number theory, it is simple to show that every numerical semigroup has a finite generating set, and in fact a unique one with minimal cardinality. The elements of this unique minimal generating system are called minimal generators, and its cardinality is known as the embedding dimension of $S$, denoted $\mathrm{e}(S)$.

Assume that $A=\left\{n_{1}, \ldots, n_{p}\right\}$ is the minimal generating set of the numerical semigroup $S$. Consider the monoid epimorphism

$$
\varphi: \mathbb{N}^{p} \rightarrow S, \quad \varphi\left(a_{1}, \ldots, a_{p}\right)=a_{1} n_{1}+\cdots+a_{p} n_{p},
$$

known as the factorization morphism of $S$. The monoid $S$ is isomorphic to $\mathbb{N}^{p} / \sigma$, where $\sigma=\left\{(a, b) \in \mathbb{N}^{p} \times \mathbb{N}^{p} \mid \varphi(a)=\varphi(b)\right\}$ is the kernel congruence of $\varphi$.

A presentation of $S$ is a system of generators of $\sigma$, and accordingly, a minimal presentation is a presentation such that none of its proper subsets generates $\sigma$.

\subsection{Numerical semigroups of embedding dimension three}

Let $S=\left\langle n_{1}, n_{2}, n_{3}\right\rangle$ be a numerical semigroup with embedding dimension three. Define $c_{i}=\min \left\{t \in \mathbb{N} \backslash\{0\} \mid t n_{i} \in\left\langle n_{j}, n_{k}\right\rangle,\{i, j, k\}=\{1,2,3\}\right\}$. Then, whenever $\{i, j, k\}=\{1,2,3\}$, there exists some $r_{i j}, r_{i k} \in \mathbb{N}$ such that

$$
c_{i} n_{i}=r_{i j} n_{j}+r_{i k} n_{k}
$$

Herzog in [18] showed that the following are equivalent: (a) $S$ is symmetric, (b) $r_{i j}=0$ for some $i, j \in\{1,2,3\}$, and (c) $S$ is a complete intersection (has a minimal presentation with two elements; see [22, Chap. 8] for details). In embedding dimension three this is also equivalent to $S$ being free, which means that it has a minimal presentation with staircase shape for some arrangement of the generators (see [22, Sec. 8.3] for the definition and characterizations of free numerical semigroup).

Theorem 2.1 ([22, Theorem 10.6]). Let $p_{1}, p_{2} \in \mathbb{N} \backslash\{0,1\}$ be relatively prime. Let $a, b$ and $c$ be nonnegative integers with $a \geq 2, b+c \geq 2$ and $\operatorname{gcd}\left(a, b p_{1}+c p_{2}\right)=1$. Then $S=\left\langle a p_{1}, a p_{2}, b p_{1}+c p_{2}\right\rangle$ is a symmetric numerical semigroup with embedding dimension three. Moreover, every embedding dimension three symmetric numerical semigroup is of this form.

\subsection{Catenary degree}

Let $S$ be minimally generated by $\left\{n_{1}, \ldots, n_{p}\right\}$. The set of factorizations of an element $n \in S$ is

$$
\mathrm{Z}(n)=\varphi^{-1}(n)=\left\{\left(a_{1}, \ldots, a_{p}\right) \in \mathbb{N}^{p} \mid a_{1} n_{1}+\cdots+a_{p} n_{p}=n\right\} .
$$


The length of a factorization $a=\left(a_{1}, \ldots, a_{p}\right) \in \mathbf{Z}(n)$ is $|a|=a_{1}+\cdots+a_{p}$. For $z=$ $\left(z_{1}, \ldots, z_{p}\right), z^{\prime}=\left(z_{1}^{\prime}, \ldots, z_{p}^{\prime}\right) \in \mathbb{N}^{p}$ write $\operatorname{gcd}\left(z, z^{\prime}\right)=\left(\min \left\{z_{1}, z_{1}^{\prime}\right\}, \ldots, \min \left\{z_{p}, z_{p}^{\prime}\right\}\right)$ and set

$$
\mathrm{d}\left(z, z^{\prime}\right)=\max \left\{\left|z-\operatorname{gcd}\left(z, z^{\prime}\right)\right|,\left|z^{\prime}-\operatorname{gcd}\left(z, z^{\prime}\right)\right|\right\}
$$

to be the distance between $z$ and $z^{\prime}$. Given $x \in \mathbb{N}^{p}$ and $Y \subset \mathbb{N}^{p}$, we define $\mathrm{d}(x, Y)=$ $\min \{d(x, y) \mid y \in Y\}$ (which exists by Dickson's Lemma; see for instance [22, Lemma 8.6]). The support of $z \in \mathbb{N}^{p}$ is defined as $\operatorname{Supp}(z)=\left\{i \in\{1, \ldots, p\} \mid z_{i} \neq 0\right\}$.

Given $n \in S, z, z^{\prime} \in \mathbf{Z}(n)$, and $N \in \mathbb{N}$, an $N$-chain of factorizations from $z$ to $z^{\prime}$ is a sequence $z_{1}, \ldots, z_{k} \in \mathrm{Z}(n)$ such that $z_{1}=z, z_{k}=z^{\prime}$ and $\mathrm{d}\left(z_{i}, z_{i+1}\right) \leq N$ for all $i$. The catenary degree of $n$, denoted $\mathrm{c}(n)$, is the minimal $N \in \mathbb{N} \cup\{\infty\}$ such that for any two factorizations $z, z^{\prime} \in \mathrm{Z}(n)$, there is an $N$-chain from $z$ to $z^{\prime}$. The catenary degree of $S$, denoted c $(S)$, is defined by

$$
\mathrm{c}(S)=\sup \{\mathrm{c}(n) \mid n \in S\}
$$

Given $z, z^{\prime} \in \mathrm{Z}(n)$ with $n \in S$, we say that $z$ and $z^{\prime}$ are $\mathcal{R}$-related if there exists a chain $z_{1}, \ldots, z_{k}$ such that

- $z_{1}=z, z_{k}=z^{\prime}$, and

- $z_{i} \cdot z_{i+1} \neq 0$ (equivalently $\operatorname{Supp}\left(z_{i}\right) \cap \operatorname{Supp}\left(z_{i+1}\right)$ is not empty) for all $i \in\{1, \ldots$, $k-1\}$.

The $\mathcal{R}$-relation is an equivalence relation and the set of $\mathcal{R}$-classes of $\mathbf{Z}(n)$ is the set of connected components of the graph $\nabla_{n}$, whose vertices are the factorizations of $n$ and $z z^{\prime}$ is an edge if $z \cdot z^{\prime} \neq 0$. This graph has the same connected components as the graph $G_{n}$ whose vertices are the minimal generators $n_{i}$ of $S$ such that $n-n_{i} \in S$, and $n_{i} n_{j}$ is an edge provided that $n-\left(n_{i}+n_{j}\right) \in S$. Since there are only finitely many elements $n$ such that the graph $G_{n}$ is disconnected (see [22, Chap. 7]), there are only finitely many $n$ for which the relation $\mathcal{R}$ yields more than one $\mathcal{R}$-class.

An element $s \in S$ is said to be a Betti element if $\mathrm{Z}(s)$ has more than one $\mathcal{R}$-class. The set of Betti elements of $S$ is denoted by $\operatorname{Betti}(S)$. Let $n \in S$ be a Betti element, and let $\mathcal{R}_{1}^{n}, \ldots, \mathcal{R}_{k_{n}}^{n}$ be the distinct $\mathcal{R}$-classes of $\mathbf{Z}(n)$. Set $\mu(n)=\max \left\{r_{1}^{n}, \ldots, r_{k_{n}}^{n}\right\}$, where $r_{i}^{n}=\min \left\{|z| \mid z \in \mathcal{R}_{i}^{n}\right\}$. Define $\mu(S)=\max \{\mu(n) \mid n \in \operatorname{Betti}(S)\}$.

Theorem 2.2 ([7, Theorem 3.1]). Let $S$ be a numerical semigroup. Then

$$
\mathrm{c}(S)=\mu(S)
$$

From the proof of this result, it follows that the catenary degree of a numerical semigroup is attained by one of its Betti elements. Several papers have recently appeared in the literature that address problems involving the catenary degree on numerical semigroups (see $[5,6,19]$ ). 


\section{3. $\omega$-primality}

An element in a numerical semigroup is irreducible if it cannot be written as a sum of nonzero elements. Obviously, the only irreducible elements in a numerical semigroup are its minimal generators.

Let $S$ be a numerical semigroup minimally generated by $\left\{n_{1}, \ldots, n_{p}\right\}$. The $\omega$-primality function assigns to each element $n \in S$ the value $\omega(S, n)=m$ if $m$ is the smallest positive integer with the property that whenever $\left(\sum_{i=1}^{p} a_{i} n_{i}\right)-n \in S$ with $|a|>m\left(a=\left(a_{1}, \ldots, a_{p}\right)\right)$, there exists $b=\left(b_{1}, \ldots, b_{p}\right) \in \mathbb{N}^{p}$ with $b \leq a$ (with the usual partial ordering on $\left.\mathbb{N}^{p}\right)$ such that $\left(\sum_{i=1}^{p} b_{i} n_{i}\right)-n \in S$ and $|b| \leq m$. Note that $\omega(S, n)<\infty$ for each $n$ in a numerical semigroup (see [16]). When $S$ is clear from the context, we simply write $\omega(n)$. Furthermore, we set

$$
\omega(S)=\sup \left\{\omega\left(S, n_{i}\right) \mid i \in\{1, \ldots, p\}\right\} .
$$

By definition, an element $b \in S$ is a prime element if and only if $\omega(S, b)=1$, and $S$ is factorial if and only if $\omega(S)=1$. Numerical semigroups other than $\mathbb{N}$ have no prime elements.

We say that $a=\left(a_{1}, \ldots, a_{p}\right) \in \mathbb{N}^{p}$ is a bullet for $n$ if $\left(\sum_{i=1}^{p} a_{i} n_{i}\right)-n \in S$ and, whenever $a_{j} \neq 0,\left(\sum_{i=1}^{p} a_{i} n_{i}\right)-n_{j}-n \notin S$. Bullets correspond to minimal elements with respect to $\leq$ in the set of all factorizations of elements of $n+S$. The set of bullets for $n \in S$ is denoted $\mathcal{B}(n)$. In numerical semigroups, the set of bullets for a fixed element $n$ lies in a bounded subset of $\mathbb{N}^{p}$.

Lemma 2.3 ([21, Lemma 3.7]). Fix a numerical semigroup $S$ minimally generated by $\left\{n_{1}, \ldots, n_{p}\right\}$ and $n \in S$.

(1) For every $i$ with $1 \leq i \leq p$, there exists $b_{i}>0$ such that $b_{i} \mathbf{e}_{i}$ is a bullet for $n$, where $\mathbf{e}_{i}$ is the ith row of the $p \times p$ identity matrix.

(2) $\mathcal{B}(n) \subseteq\left\{a \in \mathbb{N}^{p} \mid a \leq b\right\}$, where $b=\left(b_{1}, \ldots, b_{p}\right)$.

From the above lemma, for every $i \in\{1, \ldots, p\}$, we define $\mathrm{m}_{i}(n)=b_{i}$ when $n \in S$. The following proposition, which is a particular instance of $[4$, Proposition $3.3]$, is useful for computing the $\omega$ invariant.

Proposition 2.4. Let $S$ be a numerical semigroup. For every $s \in S$ we have

$$
\omega(s)=\sup \{|x| \mid x \in \mathcal{B}(n)\} .
$$

\section{Bullets and $\mathcal{R}$-Classes}

We begin with a general result on bullets.

Lemma 3.1. Let $S$ be a numerical semigroup minimally generated by = $\left\{n_{1}, \ldots, n_{p}\right\}$. Let $n \in \operatorname{Betti}(S)$ with $a, b \in \mathbf{Z}(n)$ in different $\mathcal{R}$-classes. For every $i \in \operatorname{Supp}(b)$ it follows that $a \in \mathcal{B}\left(n_{i}\right)$. 
Proof. Assume to the contrary that there exists $c \in \mathbf{Z}\left(n_{i}+S\right)$ and $x \in \mathbb{N}^{k} \backslash\{0\}$ such that $c+x=a$. From $c<a, a \cdot b=0$, and $i \in \operatorname{Supp}(b)$, we deduce that $i \notin \operatorname{Supp}(c)$. As $c \in \mathbf{Z}\left(n_{i}+S\right)$, there exists $d \in \mathbf{Z}\left(n_{i}+S\right)$ with $i \in \operatorname{Supp}(d)$ and $\varphi(c)=\varphi(d)$. Hence $\varphi(d+x)=\varphi(c+x)=\varphi(a)$.

As $0 \neq x$ and $c+x=a$, we deduce $(d+x) \cdot(c+x)=(d+x) \cdot a \neq 0$. Also, $i \in \operatorname{Supp}(b) \cap \operatorname{Supp}(d)$, and consequently $(d+x) \cdot b \neq 0$. This leads to $a \mathcal{R} b$, a contradiction.

With this Lemma and Proposition 2.4, we recover the well known equality $\mathrm{c}(S) \leq \omega(S)$ for numerical semigroups (see [17]). Lemma 3.1 has another direct consequence in embedding dimension three. Let $S=\left\langle n_{1}, n_{2}, n_{3}\right\rangle$ be a numerical semigroup with embedding dimension three. Let $c_{i}$ and $r_{j k}$ be as in (1). According to $\left[22\right.$, Example 8.23], $\operatorname{Betti}(S)=\left\{c_{1} n_{1}, c_{2} n_{2}, c_{3} n_{3}\right\}$ (though it might be the case that $c_{i} n_{i}=c_{j} n_{j}$ for some $\left.i \neq j\right)$.

Corollary 3.2. If $S=\left\langle n_{1}, n_{2}, n_{3}\right\rangle$ is a numerical semigroup with embedding dimension three, then for every $i \in\{1,2,3\}$ it follows that $\mathrm{Z}\left(c_{i} n_{i}\right) \backslash\left\{c_{i} \mathbf{e}_{i}\right\} \subseteq \mathcal{B}\left(n_{i}\right)$. Also, if $r_{i j} \neq 0$ for some $i, j \in\{1,2,3\}$ with $i \neq j$, then $c_{i} \mathbf{e}_{i} \in \mathcal{B}\left(n_{j}\right)$.

Proof. If for all $i, j, r_{i j} \neq 0$, from [4, Proposition 5.5; 12, Corollary 5], Z $\left(c_{i} n_{i}\right)=$ $\left\{c_{i} \mathbf{e}_{i}, r_{i j} \mathbf{e}_{j}+r_{i k} \mathbf{e}_{k}\right\}$. The assertion now follows from Lemma 3.1. If to the contrary $r_{i j}=0$, for some $i, j$, then we use 2 in $[14, \operatorname{Lemma} 11]$ when $\# \operatorname{Betti}(S)=2$, or $[13$, Proposition 1b)] if \# $\operatorname{Betti}(S)=1$.

We now focus on the case where $S$ is non-symmetric with embedding dimension three. Let $c_{i}, r_{i j}$ be as in Sec. 2.1. For such an $S$, it follows that $r_{i j} \neq 0$ for all $i, j \in\{1,2,3\}$. Also from [4, Proposition 5.5; 12, Corollary 5] \#Z $\left(c_{i} n_{i}\right)=2$ and, more precisely,

$$
\mathrm{Z}\left(c_{i} n_{i}\right)=\left\{c_{i} \mathbf{e}_{i}, r_{i j} \mathbf{e}_{j}+r_{i k} \mathbf{e}_{k}\right\}
$$

Proposition 3.3. Let $S=\left\langle n_{1}, n_{2}, n_{3}\right\rangle$ be a nonsymmetric numerical semigroup with embedding dimension three. Whenever $\{i, j, k\}=\{1,2,3\}$, we have that

$$
\mathcal{B}\left(n_{i}\right)=\left\{\mathbf{e}_{i}, c_{j} \mathbf{e}_{j}, c_{k} \mathbf{e}_{k}, r_{i j} \mathbf{e}_{j}+r_{i k} \mathbf{e}_{k}\right\} .
$$

Proof. The inclusion $\left\{\mathbf{e}_{i}, c_{j} \mathbf{e}_{j}, c_{k} \mathbf{e}_{k}, r_{i j} \mathbf{e}_{j}+r_{i k} \mathbf{e}_{k}\right\} \subseteq \mathcal{B}\left(n_{i}\right)$ is a consequence of Corollary 3.2. This also implies that no other element with support of cardinality one belongs to $\mathcal{B}\left(n_{i}\right)$, and that $i$ does not belong to the support of any bullet other than $\mathbf{e}_{i}$. Assume that there exists $x \mathbf{e}_{j}+y \mathbf{e}_{k} \in \mathcal{B}\left(n_{i}\right)$, with $x, y \in \mathbb{N} \backslash\{0\}$. Then $x n_{j}+y n_{k} \in n_{i}+S$, and consequently there exist $a, b, c \in \mathbb{N}$ with $a \neq 0$ such that $x n_{j}+y n_{k}=a n_{i}+b n_{j}+c n_{k}$. The minimality of $x \mathbf{e}_{i}+y \mathbf{e}_{j}$ (this element is a bullet), forces $b=c=0$. Hence $a \geq c_{i}$. Notice that $a=c_{i}$ implies $(x, y)=\left(r_{i j}, r_{i k}\right)$, since $\mathbf{Z}\left(c_{i} n_{i}\right)=\left\{c_{i} \mathbf{e}_{i}, r_{i j} \mathbf{e}_{j}+r_{i k} \mathbf{e}_{k}\right\}$. If $a>c_{i}$, then $\left(a-c_{i}\right) n_{i}+r_{i j} n_{j}+r_{i k} n_{k}=x n_{j}+y n_{k}$, 
contradicting the minimality of $x \mathbf{e}_{i}+y \mathbf{e}_{j}$, because $r_{i j} \neq 0 \neq r_{i k}$. Hence we have an equality.

From Proposition 3.3, we deduce this important corollary.

Corollary 3.4. Assume that $S=\left\langle n_{1}, n_{2}, n_{3}\right\rangle$ is a nonsymmetric numerical semigroup with embedding dimension three and with $n_{1}<n_{2}<n_{3}$. Then $\omega\left(n_{1}\right) \leq \omega\left(n_{3}\right)$.

Proof. According to Propositions 3.3 and 2.4, $\omega\left(n_{1}\right)=\max \left\{c_{2}, c_{3}, r_{12}+r_{13}\right\}$ and $\omega\left(n_{3}\right)=\max \left\{c_{1}, c_{2}, r_{31}+r_{32}\right\}$. Notice also that $c_{1}>r_{12}+r_{13}$ and $c_{3}<r_{31}+r_{32}$. The proof now follows by considering the possible values of $\omega\left(n_{3}\right)$.

- If $\omega\left(n_{3}\right)=c_{1}$, then $c_{1} \geq c_{2}$ and $c_{1} \geq r_{31}+r_{32}>c_{3}$. Also $c_{1}>r_{12}+r_{13}$, and thus we obtain $c_{1} \geq \omega\left(n_{1}\right)$.

- If $\omega\left(n_{3}\right)=c_{2}$, then $c_{2} \geq c_{1}>r_{12}+r_{13}$ and $c_{2} \geq r_{31}+r_{32}>c_{3}$. Hence $c_{2} \geq \omega\left(n_{1}\right)$.

- If $\omega\left(n_{3}\right)=r_{31}+r_{32}$, then $r_{31}+r_{32} \geq c_{1}>r_{12}+r_{13}, r_{31}+r_{32} \geq c_{2}$ and $r_{31}+r_{32}>c_{3}$. Thus, $r_{31}+r_{32} \geq \omega\left(n_{1}\right)$.

As an immediate consequence of the corollary we get the following nice result.

Corollary 3.5. Let $S=\left\langle n_{1}, n_{2}, n_{3}\right\rangle$ be a numerical semigroup with embedding dimension three and with $n_{1}<n_{2}<n_{3}$. If $\omega\left(n_{1}\right)>\omega\left(n_{3}\right)$, then $S$ is symmetric.

\section{The Symmetric Case}

Now, we wish to compute the $\omega$-values of the generators in any symmetric numerical semigroup of embedding dimension three. To do so, we will divide the claim into two cases based on the values of $b$ and $c$ in $\left\langle a p_{1}, a p_{2}, b p_{1}+c p_{2}\right\rangle$ as illustrated in Theorem 2.1. In particular $n_{1}=a p_{1}, n_{2}=a p_{2}, n_{3}=b p_{1}+c p_{2}$, with $a, b, c \in \mathbb{N}$, $a \geq 2, b \geq 2$ and $\operatorname{gcd}\left(p_{1}, p_{2}\right)=\operatorname{gcd}\left(a, n_{3}\right)=1$.

It is easy to see that $c_{1}=p_{2}, c_{2}=p_{1}, c_{3}=a$, and that minimal presentation for $S$ is given by $\left\{\left(\left(p_{2}, 0,0\right),\left(0, p_{1}, 0\right)\right),((b, c, 0),(0,0, a))\right\}$ (this is because $S=a\left\langle p_{1}+\right.$ $\left.p_{2}\right\rangle+\left(b p_{1}+c p_{2}\right) \mathbb{N}$ is a gluing of $\left\langle p_{1}, p_{2}\right\rangle$ and $\mathbb{N}$; see the proof of $[22$, Theorem 10.6]). As a consequence of Corollary 3.2, we obtain $p_{2} \mathbf{e}_{1} \in \mathcal{B}\left(a p_{2}\right)$ and $p_{1} \mathbf{e}_{2} \in \mathcal{B}\left(a p_{1}\right)$. Thus we obtain the following result.

Corollary 4.1. Let $S=\left\langle n_{1}, n_{2}, n_{3}\right\rangle$ be an embedding dimension three symmetric numerical semigroup. Then $m_{j}\left(a p_{i}\right)=p_{i}$ for $\{i, j\}=\{1,2\}$.

\subsection{The case when $S$ is representable as $\left\langle a p_{1}, a p_{2}, b p_{1}+c p_{2}\right\rangle$ with $b c \neq 0$}

In this setting, we can again use Corollary 3.2 to obtain $a \mathrm{e}_{3} \in \mathcal{B}\left(n_{1}\right) \cap \mathcal{B}\left(n_{2}\right)$. Hence, we obtain the following consequence. 
Corollary 4.2. Suppose that $S=\left\langle n_{1}, n_{2}, n_{3}\right\rangle$ is a symmetric numerical semigroup that is representable as $\left\langle a p_{1}, a p_{2}, b p_{1}+c p_{2}\right\rangle$ with $b c \neq 0$. Then $m_{3}\left(a p_{1}\right)=$ $m_{3}\left(a p_{2}\right)=a$.

Lemma 4.3. Suppose that $S=\left\langle n_{1}, n_{2}, n_{3}\right\rangle$ is a symmetric numerical semigroup that is representable as $\left\langle a p_{1}, a p_{2}, b p_{1}+c p_{2}\right\rangle$ with $b c \neq 0$.

(a) $\mathcal{B}\left(a p_{1}\right)=\left\{(1,0,0),\left(0, p_{1}, 0\right),(0,0, a)\right\}$.

(b) $\mathcal{B}\left(a p_{2}\right)=\left\{\left(p_{2}, 0,0\right),(0,1,0),(0,0, a)\right\}$.

Proof. We already know that $\left\{(1,0,0),\left(0, p_{1}, 0\right),(0,0, a)\right\} \subseteq \mathcal{B}\left(a p_{1}\right)$. Assume that $(0, x, y) \in \mathcal{B}\left(a p_{1}\right) \backslash\left\{(1,0,0),\left(0, p_{1}, 0\right),(0,0, a)\right\}$. Hence $x a p_{1}+y\left(b p_{1}+c p_{2}\right)=$ $u a p_{1}+v a p_{2}+w\left(b p_{1}+c p_{2}\right)$ for some $u, v, w \in \mathbb{N}$, with $u \neq 0$. As in the proof of Corollary 3.3, the minimality of $(0, x, y)$ implies that $v=w=0$, and yap $_{1}=$ $x a p_{1}+y\left(b p_{1}+c p_{2}\right)$. In particular, $a \mid y\left(b p_{1}+c p_{2}\right)$, and as $\operatorname{gcd}\left(a, n_{3}\right)=1$, it follows that $a \mid y$. This forces $a \leq y$, whence $(0,0, a) \leq(0, x, y)$ which is a contradiction.

The proof of $\mathcal{B}\left(a p_{2}\right)=\left\{\left(p_{2}, 0,0\right),(0,1,0),(0,0, a)\right\}$ follows in the same way.

Now, we wish to prove similar results for $b p_{1}+c p_{2}$.

Lemma 4.4. Suppose that $S=\left\langle n_{1}, n_{2}, n_{3}\right\rangle$ is a symmetric numerical semigroup that is representable as $\left\langle a p_{1}, a p_{2}, b p_{1}+c p_{2}\right\rangle$ with $b c \neq 0$. If

$$
\bar{b}=\max \left\{x \in \mathbb{N} \backslash\{0\} \mid b p_{1}+c p_{2}=x p_{1}+y p_{2}, \text { for some } y \in \mathbb{N} \backslash\{0\}\right\},
$$

and

$$
\bar{c}=\max \left\{y \in \mathbb{N} \backslash\{0\} \mid b p_{1}+c p_{2}=x p_{1}+y p_{2}, \text { for some } x \in \mathbb{N} \backslash\{0\}\right\},
$$

then

(a) $m_{1}\left(b p_{1}+c p_{2}\right)=p_{2}+\bar{b}$ and

(b) $m_{2}\left(b p_{1}+c p_{2}\right)=p_{1}+\bar{c}$.

Proof. (a) Note that the set $\left\{x \in \mathbb{N} \backslash\{0\} \mid b p_{1}+c p_{2}=x p_{1}+y p_{2}\right.$, for some $y \in$ $\mathbb{N} \backslash\{0\}\}$ is not empty (and finite) since $b$ and $c$ are both nonzero. Let $c^{\prime} \in \mathbb{N}$ such that $b p_{1}+c p_{2}=\bar{b} p_{1}+c^{\prime} p_{2}$. Then

$$
\begin{aligned}
\left(p_{2}+\bar{b}\right)\left(a p_{1}\right)-\left(b p_{1}+c p_{2}\right) & =a p_{1} p_{2}+a\left(\bar{b} p_{1}\right)-\left(b p_{1}+c p_{2}\right) \\
& =a p_{1} p_{2}+a\left(b p_{1}+c p_{2}-c^{\prime} p_{2}\right)-\left(b p_{1}+c p_{2}\right) \\
& =(a-1)\left(b p_{1}+c p_{2}\right)+\left(p_{1}-c^{\prime}\right)\left(a p_{2}\right) .
\end{aligned}
$$

Note that if $c^{\prime}>p_{1}$, then

$$
b p_{1}+c p_{2}=\bar{b} p_{1}+c^{\prime} p_{2}=\bar{b} p_{1}+\left(c^{\prime}-p_{1}\right) p_{2}+p_{1} p_{2}=\left(\bar{b}+p_{2}\right) p_{1}+\left(c^{\prime}-p_{1}\right) p_{2}
$$


contradicting the maximality of $\bar{b}$. Therefore, $\left(p_{2}+\bar{b}\right)\left(a p_{1}\right)-\left(b p_{1}+c p_{2}\right)=(a-1)$ $\left(b p_{1}+c p 2\right)+\left(p_{1}-c^{\prime}\right)\left(a p_{2}\right) \in S$ since $p_{1} \geq c^{\prime}$.

Now, note that if

$$
\begin{aligned}
\left(p_{2}+\bar{b}-1\right)\left(a p_{1}\right)-\left(b p_{1}+c p_{2}\right) & =\left(p_{2}-1\right) a p_{1}+a\left(\bar{b} p_{1}\right)-b p_{1}-c p_{2} \\
& =\left(p_{2}-1\right) a p_{1}+a\left(b p_{1}+c p_{2}-c^{\prime} p_{2}\right)-b p_{1}-c p_{2} \\
& =(a-1)\left(b p_{1}+c p_{2}\right)+a\left(p_{1} p_{2}-c^{\prime} p_{2}-p_{1}\right)
\end{aligned}
$$

is in $S$, then $(a-1)\left(b p_{1}+c p_{2}\right)+a\left(p_{1} p_{2}-p_{1}-p_{2}\right)=\left(p_{2}+\bar{b}-1\right)\left(a p_{1}\right)-\left(b p_{1}+c p_{2}\right)+$ $\left(c^{\prime}-1\right) p_{2} \in S$. However $\left[22\right.$, Remark 10.7] states that $\mathrm{F}(S)=(a-1)\left(b p_{1}+c p_{2}\right)+$ $a\left(p_{1} p_{2}-p_{1}-p_{2}\right)$, a contradiction. Therefore, $\left(p_{2}+\bar{b}-1\right)\left(a p_{1}\right)-\left(b p_{1}+c p_{2}\right) \notin S$, so $m_{1}\left(b p_{1}+c p_{2}\right)=p_{2}+\bar{b}$.

The proof of (b) is similar.

Finally, we will find $\mathcal{B}\left(b p_{1}+c p_{2}\right)$. Recall that, in our setting, a minimal presentation for $S$ is $\left\{\left(\left(p_{2}, 0,0\right),\left(0, p_{1}, 0\right)\right),((b, c, 0),(0,0, a))\right\}$, and as $b c \neq 0$, the number of Betti elements of $S$ is two. It is easy to deduce (see for instance [14, Lemma 11]) that the set of $\mathcal{R}$-classes of $\mathbf{Z}\left(a\left(b p_{1}+c p_{2}\right)\right)$ is $\left\{\{(0,0, a)\}, \mathbf{Z}\left(a\left(b p_{1}+c p_{2}\right)\right) \backslash\{(0,0, a)\}\right\}$. The second class equals $\left\{(x, y, 0) \in \mathbb{N}^{3} \mid a\left(b p_{1}+c p_{2}\right)=x a p_{1}+y a p_{2}\right\}=\{(x, y, 0) \in$ $\left.\mathbb{N}^{3} \mid b p_{1}+c p_{2}=x p_{1}+y p_{2}\right\}$.

Lemma 4.5. Suppose that $S=\left\langle n_{1}, n_{2}, n_{3}\right\rangle$ is a symmetric numerical semigroup that is representable as $\left\langle a p_{1}, a p_{2}, b p_{1}+c p_{2}\right\rangle$ with $b c \neq 0$. Then

$$
\begin{aligned}
\mathcal{B}\left(b p_{1}+c p_{2}\right)= & \left\{\left(\mathrm{m}_{1}\left(b p_{1}+c p_{2}\right), 0,0\right),\left(0, \mathrm{~m}_{2}\left(b p_{1}+c p_{2}\right), 0\right),(0,0,1)\right\} \\
& \cup\left\{(x, y, 0) \in \mathbb{N}^{3} \mid b p_{1}+c p_{2}=x p_{1}+y p_{2}, x y \neq 0\right\} .
\end{aligned}
$$

Proof. () : This inclusion is a direct consequence of Lemma 3.1 and the definition of $m_{i}$.

$(\subseteq)$ : Now, suppose that $(x, y, z) \in \mathcal{B}\left(b p_{1}+c p_{2}\right)$. If $z \neq 0, x=0$ or $y=0$, then we are done. Therefore we may now assume that $(x, y, 0) \in \mathcal{B}\left(b p_{1}+c p_{2}\right)$ with $x \neq 0$ and $y \neq 0$. Then $x\left(a p_{1}\right)+y\left(a p_{2}\right)-\left(b p_{1}+c p_{2}\right) \in S$, but $(x-1)\left(a p_{1}\right)+y\left(a p_{2}\right)-\left(b p_{1}+c p_{2}\right) \notin$ $S$ and $x\left(a p_{1}\right)+(y-1)\left(a p_{2}\right)-\left(b p_{1}+c p_{2}\right) \notin S$. Therefore, there exists $d \in \mathbb{N}$ such that $x\left(a p_{1}\right)+y\left(a p_{2}\right)-\left(b p_{1}+c p_{2}\right)=d\left(b p_{1}+c p_{2}\right)$. Since $\operatorname{gcd}\left(a, b p_{1}+c p_{2}\right)=1$ and $a\left|(d+1)\left(b p_{1}+c p_{2}\right), a\right| d+1$. Hence, there exists $d_{0} \in \mathbb{N}$ such that $d+1=a d_{0}$ and so $x p_{1}+y p_{2}=d_{0}\left(b p_{1}+c p_{2}\right)$. Note that if $d_{0} \geq 2$, then

$$
\begin{aligned}
& (x-1)\left(a p_{1}\right)+y\left(a p_{2}\right)-\left(b p_{1}+c p_{2}\right)=d\left(b p_{1}+c p_{2}\right)-a p_{1} \\
& =\left(a d_{0}-1\right)\left(b p_{1}+c p_{2}\right)-a n_{1}=(a-1)\left(b p_{1}+c p_{2}\right)+\left(d_{0}-1\right) a\left(b p_{1}+c p_{2}\right)-a p_{1} \\
& \quad=(a-1)\left(b p_{1}+c p_{2}\right)+\left(b d_{0}-b-1\right)\left(a p_{1}\right)+\left(c d_{0}-c\right)\left(a p_{2}\right) \in S
\end{aligned}
$$

since $b d_{0}-b-1 \geq 2 b-b-1=b-1 \geq 0$. This contradicts $(x-1)\left(a p_{1}\right)+y\left(a p_{2}\right)-$ $\left(b p_{1}+c p_{2}\right) \notin S$. Then $d_{0}=1$, and consequently $x p_{1}+y p_{2}=b p_{1}+c p_{2}$. 
Theorem 4.6. Suppose that $S=\left\langle n_{1}, n_{2}, n_{3}\right\rangle$ is a symmetric numerical semigroup that is representable as $\left\langle a p_{1}, a p_{2}, b p_{1}+c p_{2}\right\rangle$ with $b c \neq 0$. Let

$$
\bar{b}=\max \left\{x \in \mathbb{N} \backslash\{0\} \mid b p_{1}+c p_{2}=x p_{1}+y p_{2}, \text { for some } y \in \mathbb{N} \backslash\{0\}\right\},
$$

and

$$
\bar{c}=\max \left\{y \in \mathbb{N} \backslash\{0\} \mid b p_{1}+c p_{2}=x p_{1}+y p_{2}, \text { for some } x \in \mathbb{N} \backslash\{0\}\right\},
$$

then

(a) $\omega\left(a p_{1}\right)=\max \left(a, p_{1}\right)$

(b) $\omega\left(a p_{2}\right)=\max \left(a, p_{2}\right)$, and

(c) $\omega\left(b p_{1}+c p_{2}\right)=\max \left(\bar{b}+p_{2}, \bar{c}+p_{1}\right)$.

Proof. The assertions (a) and (b) follow directly from Proposition 2.4 and Lemma 4.3. For (c), we will divide the claim into two cases.

First suppose that $p_{1}<p_{2}$. Let $c^{\prime} \in \mathbb{N}$ such that $\bar{b} p_{1}+c^{\prime} p_{2}=b p_{1}+c p_{2}$ and let $(x, y, 0) \in \mathbb{N}^{3}$ with $b p_{1}+c p_{2}=x p_{1}+y p_{2}$. Then $(\bar{b}-x) p_{1}=\left(y-c^{\prime}\right) p_{2}$, and the maximality of $\bar{b}$ implies $\bar{b}-x \geq 0$. As $\operatorname{gcd}\left(p_{1}, p_{2}\right)=1, p_{2} \mid(\bar{b}-x)$. This means that $x=\bar{b}-d p_{2}$ for some $d \in \mathbb{N}_{0}$. Therefore $y=c^{\prime}+d p_{1}$, since $\left(y-c^{\prime}\right) p_{2}=$ $(\bar{b}-x) p_{1}=d p_{2} p_{1}$. As a result, we see that $x+y=\bar{b}-d p_{2}+c^{\prime}+d p_{1} \leq \bar{b}+c^{\prime}$, since $p_{1}<p_{2}$. However, in the proof of Lemma 4.4, we noted that $c^{\prime} \leq p_{1}$. Consequently, $x+y \leq \bar{b}+c^{\prime} \leq \bar{b}+p_{1}<\bar{b}+p_{2}$. By Proposition 2.4, Lemmas 4.4 and 4.5 , the result follows.

The proof for $p_{1}>p_{2}$ is analogous.

Observe that we have not given a closed formula for (c) in the last theorem in terms of $a, b, c, p_{1}$ and $p_{2}$. As $p_{1}$ and $p_{2}$ are coprime, $\left\langle p_{1}, p_{2}\right\rangle$ is a numerical semigroup. Its minimal presentation is $\left\{\left(\left(p_{2}, 0\right),\left(0, p_{1}\right)\right)\right\}$. Then whenever $k \in \mathbb{N}$ is such that $c-k p_{1} \in \mathbb{N}$, the element $\left(b+k p_{2}\right) p_{1}+\left(c-k p_{1}\right) p_{2} \in \mathbf{Z}\left(b p_{1}+c p_{2}\right)$. The same holds if $b-k p_{2} \in \mathbb{N}$, which yields $\left(b-k p_{2}\right) p_{1}+\left(c+k p_{1}\right) p_{2} \in \mathbf{Z}\left(b p_{1}+c p_{2}\right)$. The largest $k$ such that $c-k p_{1} \in \mathbb{N} \backslash\{0\}$ is $c / p_{1}-1$ if $p_{1}$ divides $c$, and $\left\lfloor\frac{c}{p_{1}}\right\rfloor$ otherwise. A similar argument applies for the largest $k$ fulfilling $b-k p_{2} \in \mathbb{N} \backslash\{0\}$. From this we deduce that

$$
\begin{aligned}
& \bar{b}= \begin{cases}b+\left(\frac{c}{p_{1}}-1\right) p_{2} & \text { if } c \bmod p_{1}=0, \\
b+\left\lfloor\frac{c}{p_{1}}\right\rfloor p_{2} & \text { otherwise, }\end{cases} \\
& \bar{c}= \begin{cases}c+\left(\frac{b}{p_{2}}-1\right) p_{1} & \text { if } b \bmod p_{2}=0, \\
c+\left\lfloor\frac{b}{p_{2}}\right\rfloor p_{1} & \text { otherwise. }\end{cases}
\end{aligned}
$$




\subsection{The case when $S$ is not representable as $\left\langle a p_{1}, a p_{2}, b p_{1}+c p_{2}\right\rangle$ with $b c \neq 0$}

Recall from Theorem 2.1 that $b+c \geq 2$. Thus the hypothesis that $b c=0$ implies that exactly one of $b$ or $c$ is zero. Without loss of generality, suppose that $c=0$. Then $S=\left\langle a p_{1}, a p_{2}, b p_{1}\right\rangle$. If $b>p_{2}$, then $b p_{1}=\left(b-p_{2}\right) n_{1}+\left(p_{1}\right) n_{2}$, contradicting the fact that $S$ is not representable in the form above with $b \neq 0$ and $c \neq 0$. Therefore, $b \leq p_{2}$. Note that $\operatorname{gcd}(a, b)=1$ and $\operatorname{gcd}\left(a, p_{1}\right)=1$ since $\operatorname{gcd}\left(a, b p_{1}\right)=1$. As a result, we see that $S=\left\langle p_{1} a, p_{1} b, p_{2} a\right\rangle$ is of the form in Theorem $2.1 \operatorname{since} \operatorname{gcd}\left(p_{1}, p_{2} a\right)=$ 1 and $\operatorname{gcd}(a, b)=1$. Note that $p_{2} \leq b$ since otherwise, $p_{2} a=\left(p_{2}-b\right) a+a b$, contradicting the fact that $S$ is not representable in the above form with $b \neq 0$ and $c \neq 0$. Therefore, $b=p_{2}$, so $S=\left\langle a p_{1}, a p_{2}, p_{1} p_{2}\right\rangle$, where $a, p_{1}$, and $p_{2}$ are pairwise relatively prime.

Throughout the rest of the section, let $S=\langle a b, a c, b c\rangle$, where $a, b, c$ are pairwise relatively prime integers greater than one. We write $n_{1}=a b, n_{2}=a c$ and $n_{3}=b c$.

If we find the $\omega$-values of the generators of $S$, then by our discussion at the beginning of this section, this will complete our characterization of the $\omega$-values of the generators for any symmetric numerical semigroup of embedding dimension three. Moreover, Theorem 4.9 will also cover the case when $S$ is nonsymmetric.

According to [13, Theorem 12], $\operatorname{Betti}(S)=\{a b c\}$, and by [13, Proposition 1], each $\mathcal{R}$-class in $\mathbf{Z}(a b c)$ is a singleton. Thus, we deduce that the three $\mathcal{R}$-classes of $\mathbf{Z}(a b c)$ are $\{(c, 0,0)\},\{(0, b, 0)\}$ and $\{(0,0, a)\}$. As a direct consequence of Lemma 3.1, we get that $(0,0, a) \in \mathcal{B}\left(n_{1}\right) \cap \mathcal{B}\left(n_{2}\right),(0, b, 0) \in \mathcal{B}\left(n_{1}\right) \cap \mathcal{B}\left(n_{3}\right)$ and $(c, 0,0) \in \mathcal{B}\left(n_{2}\right) \cap \mathcal{B}\left(n_{3}\right)$. In particular, we obtain the following consequence.

Corollary 4.7. If $S=\langle a b, a c, b c\rangle$, with $a, b, c$ pairwise relatively prime integers greater than one, then we have the following.

(a) $m_{1}(a c)=m_{1}(b c)=c$.

(b) $m_{2}(a b)=m_{2}(b c)=b$.

(c) $m_{3}(a b)=m_{3}(a c)=a$.

Lemma 4.8. Let $S=\langle a b, a c, b c\rangle$, with $a, b, c$ pairwise relatively prime integers greater than one.

(a) $\mathcal{B}(a b)=\{(1,0,0),(0, b, 0),(0,0, a)\}$.

(b) $\mathcal{B}(a c)=\{(c, 0,0),(0,1,0),(0,0, a)\}$.

(c) $\mathcal{B}(a b)=\{(c, 0,0),(0, b, 0),(0,0,1)\}$.

Proof. From Corollary 3.2, we already know that the inclusion $\{(1,0,0),(0, b, 0)$, $(0,0, a)\} \subseteq \mathcal{B}(a b)$ holds. Assume that $(0, x, y) \in \mathcal{B}(a b) \backslash\{(0, b, 0),(0,0, a)\}$. Then $x a c+y b c=u a b+v a c+w b c$ for some $u, v, w \in \mathbb{N}$ with $u \neq 0$. The minimality of $(0, x, y)$ forces $v=w=0$. Hence $u a b=x a c+y b c$. Thus $b \mid x a c$ and $a \mid y b c$. Since $a$, $b$ and $c$ are pairwise prime, this means that $b \mid x$ and $a \mid y$. If $x \neq 0$, then $b<x$, and 
$(0, b, 0)<(0, x, y)$, contradicting the minimality of $(0, x, y)$. The same if $y \neq 0$. In both cases we get a contradiction.

The proofs of (b) and (c) are similar to that of (a).

We now state a theorem that gives the $\omega$-values of the generators of the numerical semigroup $S=\langle a b, a c, b c\rangle$. The proof follows immediately from Proposition 2.4 and Lemma 4.8 .

Theorem 4.9. Let $S=\langle a b, a c, b c\rangle$, with $a, b, c$ pairwise relatively prime integers greater than one.

(a) $\omega(a b)=\max (a, b)$.

(b) $\omega(a c)=\max (a, c)$.

(c) $\omega(b c)=\max (b, c)$.

Together, Theorems 4.6 and 4.9 give the $\omega$-values of the generators for any symmetric numerical semigroup with embedding dimension three.

\section{Comparing the $\omega$-Primality with the Catenary Degree}

Let $S=\left\langle n_{1}, n_{2}, n_{3}\right\rangle$ with $n_{1}<n_{2}<n_{3}$ be a numerical semigroup with embedding dimension three. Recall that if we set $c_{i}=\min \left\{t \in \mathbb{N} \backslash\{0\} \mid t n_{i} \in\left\langle n_{j}, n_{j}\right\},\{i, j, k\}=\right.$ $\{1,2,3\}$, then $\operatorname{Betti}(S)=\left\{c_{1} n_{1}, c_{2} n_{2}, c_{3} n_{3}\right\}$, and that if $S$ is not symmetric some of these Betti elements coincide. We also know that $\mathrm{c}(S) \leq \omega(S)$.

We say that $S$ is uniquely presented if for every two minimal presentations $\tau$ and $\tau^{\prime}$ and every $(a, b) \in \tau$, either $(a, b) \in \tau^{\prime}$ or $(b, a) \in \tau^{\prime}$ (that is, the minimal presentation is unique up to rearranging the pairs in the presentation). We will make use of this concept in the proof of the following theorem. It is well known that if $S$ is not symmetric, then it is uniquely presented.

Theorem 5.1. Let $S=\left\langle n_{1}, n_{2}, n_{3}\right\rangle$ with $n_{1}<n_{2}<n_{3}$ be a numerical semigroup with embedding dimension three. For $\{i, j, k\}=\{1,2,3\}$, set $c_{i}=\min \{t \in$ $\left.\mathbb{N} \backslash\{0\} \mid t n_{i} \in\left\langle n_{j}, n_{j}\right\rangle\right\}$.

(a) If \# $\operatorname{Betti}(S)=3$, then $\omega(S)=\mathrm{c}(S)$.

(b) If $c_{1} n_{1}=c_{2} n_{2} \neq c_{3} n_{3}$, then $\mathrm{c}(S)<\omega(S)$.

(c) If $c_{1} n_{1}=c_{3} n_{3} \neq c_{2} n_{2}$, then $\mathrm{c}(S)<\omega(S)$.

(d) If $c_{1} n_{1} \neq c_{2} n_{2}=c_{3} n_{3}$ and $c_{2} n_{2} \mid c_{1} n_{1}$, then $\mathrm{c}(S)=\omega(S)$.

(e) If $c_{1} n_{1}=c_{2} n_{2}=c_{3} n_{3}$, then $\mathrm{c}(S)=\omega(S)$.

Proof. We will use $\bar{b}$ and $\bar{c}$ as defined in Theorem 4.6:

$$
\begin{aligned}
& \bar{b}=\max \left\{x \in \mathbb{N} \backslash\{0\} \mid b p_{1}+c p_{2}=x p_{1}+y p_{2}, \text { for some } y \in \mathbb{N} \backslash\{0\}\right\}, \\
& \bar{c}=\max \left\{y \in \mathbb{N} \backslash\{0\} \mid b p_{1}+c p_{2}=x p_{1}+y p_{2}, \text { for some } x \in \mathbb{N} \backslash\{0\}\right\} .
\end{aligned}
$$


(a) This is a consequence of [4, Corollary 5.8].

(b) In this setting, $n_{1}=a p_{1}, n_{2}=a p_{2}$ and $n_{3}=b p_{1}+c p_{2}$, with $p_{1}, p_{2}, a, b$ and $c$ as in Theorem 2.1. As we previously mentioned in the second paragraph of Sec. 4, it easily follows that $c_{1}=p_{2}, c_{2}=p_{1}$ and $c_{3}=a$. Notice also that $c_{1}>c_{2}$. Let $R$ be the $\mathcal{R}$-class of $\mathbf{Z}\left(c_{3} n_{3}\right)$ not containing $\left(0,0, c_{3}\right)$. From Theorem 2.2 we know that the catenary degree of $S$ is $\mathrm{c}(S)=\max \left\{c_{1}, \min \{r+s \mid(r, s, 0) \in\right.$ $R\}\}=\max \left\{p_{2}, \min \left\{b+c \mid b p_{1}+c p_{2}=n_{3}\right\}\right\}$. We also know that the $\omega$-primality of $S$ is $\omega(S)=\max \left\{a, p_{2}+\bar{b}, p_{1}+\bar{c}\right\}$ (Theorem 4.6).

Observe that if $S$ is uniquely presented we easily derive that $\bar{b}<p_{2}$ and $\bar{c}<p_{1}$. From $\bar{c}<p_{1}$ we obtain $\bar{c}<p_{1}<p_{2}$ and thus $\min \left\{b+c \mid b p_{1}+c p_{2}=\right.$ $\left.n_{3}\right\}=\bar{b}+\bar{c}<\bar{b}+p_{2}$. We have $\mathrm{c}(S)=\max \left\{p_{2}, \bar{b}+\bar{c}\right\}<\bar{b}+p_{2} \leq \omega(S)$.

If $S$ is not uniquely presented, let $c^{\prime}$ be such that $\left(\bar{b}, c^{\prime}, 0\right) \in \mathrm{Z}\left(a n_{3}\right)$ fulfills $\max \left\{b+c \mid b p_{1}+c p_{2}=n_{3}\right\}=\bar{b}+c^{\prime}$. From $\min \left\{b+c \mid b p_{1}+c p_{2}=n_{3}\right\}<$ $\max \left\{b+c \mid b p_{1}+c p_{2}=n_{3}\right\}=\bar{b}+c^{\prime}$, and $p_{2}<p_{2}+\bar{b}$, we obtain $c(S)<\omega(S)$.

(c) As in the preceding case, we obtain $n_{1}=a p_{1}, n_{3}=a p_{2}$ and $n_{2}=b p_{1}+c p_{2}$, and $c_{1}=p_{2}, c_{2}=a$ and $c_{3}=p_{1}$. Let $R$ be the $\mathcal{R}$-class of $\mathrm{Z}\left(c_{2} n_{2}\right)$ not containing $\left(0, c_{2}, 0\right)$. From Theorem 2.2 we know that the catenary degree of $S$ is c $(S)=$ $\max \left\{c_{1}, c_{2}, \min \{r+s \mid(r, 0, s) \in R\}\right\}=\max \left\{p_{2}, a, \min \left\{b+c \mid b p_{1}+c p_{2}=n_{2}\right\}\right\}$. As a consequence of Theorem 4.6, $\omega(S)=\max \left\{a, p_{2}+\bar{b}, p_{1}+\bar{c}\right\}$.

Observe that if $S$ is uniquely presented we obtain $\bar{c}<p_{1}<p_{2}$ and then $\bar{b}+\bar{c}<\bar{b}+p_{2}$. Furthermore $n_{1}=a p_{1}<n_{2}=\bar{b} p_{1}+\bar{c} p_{2}<\bar{b} p_{1}+p_{1} p_{2}=\left(\bar{b}+p_{2}\right) p_{1}$, so $\mathrm{c}(S)=\max \left\{p_{2}, a, \bar{b}+\bar{c}\right\}<\bar{b}+p_{2} \leq \omega(S)$.

If $S$ is not uniquely presented, from $\min \left\{b+c \mid b p_{1}+c p_{2}=n_{2}\right\}<\max \{b+$ $\left.c \mid b p_{1}+c p_{2}=n_{2}\right\}=\bar{b}+c^{\prime}$, with $c^{\prime}$ such that $\left(\bar{b}, 0, c^{\prime}\right) \in \mathrm{Z}\left(a n_{2}\right)$. From the proof of Lemma 4.4, we know that $c^{\prime} \leq p_{1}$, which is smaller than $p_{2}$. Also $p_{2}<p_{2}+\bar{b}$. Moreover, as in the preceding paragraph, $n_{1}=a p_{1}<\left(\bar{b}+p_{2}\right) p_{1}$ and then $a<\bar{b}+p_{2}$. Furthermore, if $b^{\prime}$ is such that $\left(b^{\prime}, 0, \bar{c}\right) \in \mathbf{Z}\left(n_{2}\right)$, we have $b^{\prime}+\bar{c}<\bar{b}+c^{\prime}<\bar{b}+p_{2}$. Thus we obtain $\mathrm{c}(S)=\max \left\{p_{2}, a, b^{\prime}+\bar{c}\right\}<\bar{b}+p_{2} \leq \omega(S)$.

(d) This is a consequence of the main result in [14].

(e) Follows from [13, Theorem 19].

Remark 5.2. In the case $c_{1} n_{1} \neq c_{2} n_{2}=c_{3} n_{3}$, we have $c_{1}=a, c_{2}=p_{2}, c_{3}=p_{1}$. We now consider several examples to illustrate that the statement of Theorem 5.1 is best possible.

We know from Theorem 2.2 that $\mathrm{c}(S)=\max \left\{c_{1}, c_{2}\right\}=\max \left\{a, p_{2}\right\}$. Observe, we have $(b+c) p_{1}<b p_{1}+c p_{2}<a p_{1}$ and then $b+c<a$. In light of Theorem 4.6, $\omega(S)=\max \left\{a, \bar{b}+p_{2}, \bar{c}+p_{1}\right\}$. So we can say that if $a \geq \max \left\{\bar{b}+p_{2}, \bar{c}+p_{1}\right\}$, then $\mathrm{c}(S)=\omega(S)=a$, while if $a<\max \left\{\bar{b}+p_{2}, \bar{c}+p_{1}\right\}$, then $\mathrm{c}(S)<\omega(S)$.

If $a n_{1} \in\left\langle n_{2}\right\rangle, \omega(S)=\max \left\{a, p_{1}+\bar{c}, \bar{b}\right\}$, then for $a \geq \max \left\{\bar{b}, \bar{c}+p_{1}\right\}$ we get $\mathrm{c}(S)=\omega(S)=a$ and otherwise $\mathrm{c}(S)<\omega(S)$. If $a n_{1} \in\left\langle n_{3}\right\rangle, \omega(S)=\max \left\{a, p_{2}+\bar{b}\right\}$ and then, for $a \geq p_{2}+\bar{b}, \mathrm{c}(S)=\omega(S)=a$, while $\mathrm{c}(S)<\omega(S)$ otherwise. We have examples of the two situations, in both cases uniquely and nonuniquely presented. The examples have been obtained by using the idea of gluing and the package [9]. 
For instance, if we want to produce an example $S=\left\langle n_{1}, n_{2}, n_{3}\right\rangle$ with $n_{1}<b_{2}<n_{3}$ and $c_{1} n_{1} \neq c_{2} n_{2}=c_{3} n_{3}$, then we do the following. We start with the semigroup generated by $T=\left\langle p_{1}, p_{2}\right\rangle$ with $p_{1}<p_{2}$ and $\operatorname{gcd}\left(p_{1}, p_{2}\right)=1$. We know that a minimal presentation for $T$ is $\left\{\left(\left(p_{2}, 0\right),\left(p_{1}, 0\right)\right)\right\}$ and $\operatorname{Betti}(T)=\left\{p_{1} p_{2}\right\}$. Next we take $a, b, c \in \mathbb{N} \backslash\{0\}$ such that $a p_{1}>b p_{1}+c p_{2}$ (and thus $b p_{1}+c p_{2}<a p_{1}<a p_{2}$ ), and $\operatorname{gcd}\left(a, b p_{1}+c p_{2}\right)=1$. Define $n_{1}=b p_{1}+b p_{2}, n_{2}=a p_{1}$ and $n_{3}=b p_{2}$. If follows that $S$ is the gluing of $T$ and $\mathbb{N}$ (see [22, Chap. 8] for the definition of gluing), and that a minimal presentation for $S$ is given by $\left\{((a, 0,0),(0, b, c)),\left(\left(0, p_{2}, 0\right),\left(0,0, p_{1}\right)\right)\right\}$ ([22, Theorem 9.2]).

(Ex 1) $S=\langle 19,350,490\rangle$ is uniquely presented and $c_{1} n_{1} \neq c_{2} n_{2}=c_{3} n_{3}$. We have $\mathrm{c}(S)=\omega(S)=70$. Here $S=(5+2 \times 7) \mathbb{N}+70\langle 5,7\rangle ; p_{1}=5, p_{2}=7, a=70$, $b=1$ and $c=2$.

(Ex 2$) S=\langle 17,40,56\rangle$ is uniquely presented and $c_{1} n_{1} \neq c_{2} n_{2}=c_{3} n_{3}$. We have $\mathrm{c}(S)=8<\omega(S)=9$.

(Ex 3$) S=\langle 75,130,234\rangle$ is not uniquely presented and $c_{1} n_{1} \neq c_{2} n_{2}=c_{3} n_{3}$. We have $\mathrm{c}(S)=\omega(S)=26$.

(Ex 4) $S=\langle 62,63,147\rangle$ is not uniquely presented and $c_{1} n_{1} \neq c_{2} n_{2}=c_{3} n_{3}$. We have $\mathrm{c}(S)=21<\omega(S)=23$.

A particular case of the situation in which $a \geq \max \left\{\bar{b}+p_{2}, \bar{c}+p_{1}\right\}$ is when $c_{2} n_{2}$ divides $c_{1} n_{1}$. Notice that $a \geq\left\{\bar{b}+p_{2}, \bar{c}+p_{1}\right\}$ is a necessary condition for $c_{2} n_{2} \mid c_{1} n_{1}$ but not sufficient, as we can see in (Ex 1). Indeed in that case $a=70 \geq \max \{8,7\}$ but $c_{2} n_{2}=7 \cdot 350$ does not divide $c_{1} n_{1}=70 \cdot 19=1330$.

\section{The Inequalities Involving the $\omega$-Values of the Generators}

As in the preceding section, let $S$ be minimally generated by $\left\{n_{1}, n_{2}, n_{3}\right\}$ with $n_{1}<$ $n_{2}<n_{3}$. As we mentioned in the Introduction, there are 13 possible inequalities involving the $\omega\left(n_{i}\right)$.

Theorem 6.1. Let $S=\left\langle n_{1}, n_{2}, n_{3}\right\rangle$ be a numerical semigroup with embedding dimension three and with $n_{1}<n_{2}<n_{3}$. When $\{i, j, k\}=\{1,2,3\}$, set $c_{i}=\min \{t \in$ $\left.\mathbb{N} \backslash\{0\} \mid t n_{i} \in\left\langle n_{j}, n_{j}\right\rangle\right\}$.

(a) If \# $\operatorname{Betti}(S)=3$, then $\omega\left(n_{1}\right) \leq \omega\left(n_{3}\right)$.

(b) If $c_{1} n_{1}=c_{2} n_{2} \neq c_{3} n_{3}$, then $\omega\left(n_{1}\right) \leq \omega\left(n_{2}\right)<\omega\left(n_{3}\right)$.

(c) If $c_{1} n_{1}=c_{3} n_{3} \neq c_{2} n_{2}$, then $\omega\left(n_{1}\right) \leq \omega\left(n_{3}\right)<\omega\left(n_{2}\right)$.

(d) If $c_{1} n_{1}=c_{2} n_{2}=c_{3} n_{3}$, then $\omega\left(n_{1}\right)<\omega\left(n_{2}\right)=\omega\left(n_{3}\right)$.

\section{Proof.}

(a) This is just Corollary 3.4.

(b) From Theorem 4.6, we get that $\omega\left(n_{1}\right)=\max \left\{a, p_{1}\right\}, \omega\left(n_{2}\right)=\max \left\{a, p_{2}\right\}$, and $\omega\left(n_{3}\right)=\max \left\{\bar{b}+p_{2}, \bar{c}+p_{1}\right\}$. Obviously, $\bar{b}+p_{2}>p_{2}$ and $\bar{c}+p_{1}>p_{1}$, so it is 
sufficient to show that $\omega\left(n_{3}\right)>a$. Notice that $a p_{2}=n_{2}<n_{3}=b^{\prime} p_{1}+\bar{c} p_{2}<$ $\left(b^{\prime}+\bar{c}\right) p_{2}$, so $a<b^{\prime}+\bar{c} \leq \omega\left(n_{3}\right)$.

(c) From Theorem 4.6, we get that $\omega\left(n_{1}\right)=\max \left\{a, p_{1}\right\}, \omega\left(n_{3}\right)=\max \left\{a, p_{2}\right\}$, and $\omega\left(n_{2}\right)=\max \left\{\bar{b}+p_{2}, \bar{c}+p_{1}\right\}$. Obviously, $\bar{b}+p_{2}>p_{2}$ and $\bar{c}+p_{1}>p_{1}$, so it is sufficient to show that $\omega\left(n_{2}\right)>a$. Notice that $a p_{1}=n_{1}<n_{2}=\bar{b} p_{1}+c^{\prime} p_{2} \leq$ $\bar{b} p_{1}+p_{1} p_{2}$, so $a<\bar{b}+p_{2} \leq \omega\left(n_{2}\right)$.

(d) By Theorem 4.9, $\omega\left(n_{1}\right)=\max \{a, b\}=b, \omega\left(n_{2}\right)=\max \{a, c\}=c$, and $\omega\left(n_{3}\right)=$ $\max \{b, c\}=c$, so we are done.

\subsection{Orderings when $\omega\left(n_{1}\right)>\omega\left(n_{3}\right)$}

Suppose that $\omega\left(n_{1}\right)>\omega\left(n_{3}\right)$. Then by Corollary $3.5, S$ is symmetric. Therefore, by Theorem 6.1, we must have $c_{1} n_{1} \neq c_{2} n_{2}=c_{3} n_{3}$ which implies, by Theorem 4.6, that $\omega\left(n_{1}\right)=\max \left\{\bar{b}+p_{2}, \bar{c}+p_{1}\right\}, \omega\left(n_{2}\right)=\max \left\{a, p_{1}\right\}$ and $\omega\left(n_{3}\right)=\max \left\{a, p_{2}\right\}$. Since $a p_{1}=n_{2}<n_{3}=a p_{2}$, this means that $\omega\left(n_{2}\right) \leq \omega\left(n_{3}\right)$. Note that $\bar{b}+p_{2}>p_{2}$ and $\bar{c}+p_{1}>p_{1}$, and thus

(1) if $a<p_{2}$, then $\omega\left(n_{2}\right)<\omega\left(n_{3}\right)<\omega\left(n_{1}\right)$,

(2) if $p_{2} \leq a<\omega\left(n_{1}\right)$, then $\omega\left(n_{2}\right)=\omega\left(n_{3}\right)<\omega\left(n_{1}\right)$,

(3) if $a \geq \omega\left(n_{1}\right)$, then $\omega\left(n_{1}\right) \leq \omega\left(n_{2}\right)=\omega\left(n_{3}\right)$.

Hence, we have verified the following conjecture from [8].

Theorem 6.2. Let $S=\left\langle n_{1}, n_{2}, n_{3}\right\rangle$ be a numerical semigroup with embedding dimension three. The sequence $\omega\left(n_{1}\right), \omega\left(n_{2}\right)$, and $\omega\left(n_{3}\right)$ does not satisfy any of the following three orderings:

- $\omega\left(n_{1}\right)>\omega\left(n_{2}\right)>\omega\left(n_{3}\right)$;

- $\omega\left(n_{1}\right)=\omega\left(n_{2}\right)>\omega\left(n_{3}\right)$;

- $\omega\left(n_{1}\right)<\omega\left(n_{2}\right), \omega\left(n_{2}\right)>\omega\left(n_{3}\right), \omega\left(n_{3}\right)<\omega\left(n_{1}\right)$.

The interested reader is directed to the tables in both $[3,8]$ for examples illustrating each of the remaining 10 possible sets of inequalities.

\section{Acknowledgment}

The first and third authors received National Science Foundation support under DMS-1262897. The second author is supported by the projects MTM2010-15595, FQM-343, FQM-5849, NSF-1061366 and FEDER funds. The authors would like to thank the referee for their comments and suggestions.

\section{References}

[1] D. F. Anderson and S. T. Chapman, How far is an element from being prime? J. Algebra Appl. 9 (2010) 1-11.

[2] D. F. Anderson and S. T. Chapman, On bounding measures of primeness in integral domains, Int. J. Algebra Comput. 22 (2012), pp. 15. 
[3] D. F. Anderson, S. T. Chapman, N. Kaplan and D. Torkornoo, An algorithm to compute $\omega$-primality in a numerical monoid, Semigroup Forum 82 (2011) 96-108.

[4] V. Blanco, P. A. García-Sánchez and A. Geroldinger, Semigroup-theoretical characterizations of arithmetical inveriants with application to numerical monoids and Krull monoids, Illinois J. Math. 55 (2011) 1385-1414.

[5] S. T. Chapman, M. Corrales, A. Miller, C. Miller and D. Patel, The catenary and tame degrees on a numerical monoid are eventually periodic, J. Australian Math. Soc. 97 (2014) 289-300.

[6] S. T. Chapman, P. A. García-Sánchez and D. Llena, The catenary and tame degree of numerical monoids, Forum Mathematicum 21 (2009) 117-129.

[7] S. T. Chapman, P. A. García-Sánchez, D. Llena, V. Ponomarenko and J. C. Rosales, The catenary and tame degree in finitely generated commutative cancellative monoids, Manuscripta Math. 120 (2006) 253-264.

[8] S. T. Chapman, W. Puckett and K. Shour, On the omega values of generators of embedding dimension three numerical monoids generated by an interval, Involve 7 (2014) 657-667.

[9] M. Delgado, P. A. García-Sánchez and J. Morais, "NumericalSgps", A GAP Package for Numerical Semigroups, available via http://www.gap-system.org.

[10] J. I. García-García and A. V. Tenorio, Omega primality, a package for computing the omega primality of finitely generated atomic monoids (2014), available at http://rodin.uca.es/xmlui/handle/10498/15961.

[11] J. I. García-García, M. A. Moreno-Frías and A. V. Tenorio, Computation of the w-primality and asymptotic w-primality, applications to numerical semigroups, Israel J. Math., to appear, arXiv preprint, arXiv:1307.5807 (2013).

[12] P. A. García-Sánchez and I. Ojeda, Uniquely presented finitely generated commutative monoids, Pacific J. Math. 248 (2010) 91-105.

[13] P. A. García-Sánchez, I. Ojeda and J. C. Rosales, Affine semigroups having a unique Betti element, J. Algebra Appl. 12 (2013), 11 pp.

[14] P. A. García-Sánchez and C. Viola, When the catenary degree meets the tame degree in embedding dimension three numerical semigroups, Involve, to appear.

[15] A. Geroldinger and F. Halter-Koch, Non-Unique Factorizations: Algebraic, Combinatorial, and Analytic Theory (Chapman and Hall/CRC, Boca Raton, Florida, 2006).

[16] A. Geroldinger and W. Hassler, Local tameness of v-Noetherian monoids, J. Pure Applied Algebra 212 (2008) 1509-1524.

[17] A. Geroldinger and F. Kainrath, On the arithmetic of tame monoids with applications to Krull monoids and Mori domains, J. Pure Appl. Algebra 214 (2010) 2199-2218.

[18] J. Herzog, Generators and relations of abelian semigroups and semigroup rings, Manuscripta Math. 3 (1970) 175-193.

[19] M. Omidali, The catenary and tame degree of numerical monoids generated by generalized arithmetic sequences, Forum Math. 24 (2012) 627-640.

[20] C. O'Neill and R. Pelayo, On the linearity of $\omega$-primality in numerical monoids, J. Pure Appl. Algebra 218 (2014) 1620-1627.

[21] C. O'Neill and R. Pelayo. How do you measure primality? Amer. Math. Monthly, to appear, arXiv:1405.1714.

[22] J. C. Rosales and P. A. García-Sánchez, Numerical semigroups, Developments in Mathematics 20, (Springer, New York, 2009). 